\title{
ESTRATEGIA PARA LA COMERCIALIZACIÓN DEL SERVICIO PREMIUM DEL HOTEL PLAYA PESQUERO
}

\section{STRATEGY FOR THE MARKETING OF THE PREMIUM SERVICE OF THE PLAYA PESQUERO HOTEL}

\author{
José L. Figueiras Rodríguez \\ Hotel Playa Pesquero \\ Rafael Freyre, Holguín, Cuba \\ iefe.riesgos@ppesquero.co.cu
}

\author{
Migdely Barbarita Ochoa Avila \\ Faculty of Business Sciences \\ University of Holguin \\ Holguín, Holguín, Cuba \\ migdely@uho.edu.cu
}

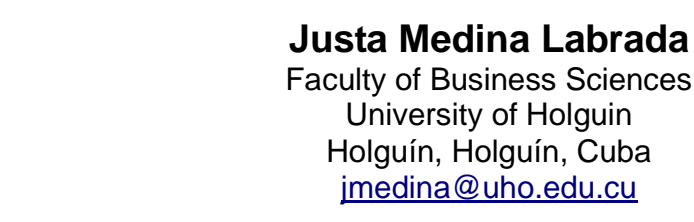

Fecha de recepción: 28/02/2019 - Fecha de aprobación: 09/05/2019

\section{RESUMEN}

La comercialización en hoteles de lujo es relevante para satisfacer las demandas crecientes de clientes, en ello, los servicios Premium son fundamentales para mantener los ingresos planificados. La investigación definió como problema científico el siguiente: La insuficiente comercialización desde lo estratégico del servicio Premium, limita los ingresos de este servicio en el hotel Playa Pesquero. Se trazó como objetivo general: Desarrollar una estrategia para la comercialización del servicio Premium, que contribuya a incrementar los ingresos de este servicio en el hotel Playa Pesquero. Se emplearon varios métodos teóricos como: análisis y síntesis, inductivo-deductivo, sistémico estructural, y entre los empíricos: la observación científica, entrevista, la revisión de documentos y el criterio de especialistas. La estrategia para la comercialización del servicio Premium se estructuró en cuatro componentes esenciales: objetivos, fases, tareas, y técnicas a emplear. La aplicación parcial de la estrategia ha logrado entre sus resultados los siguientes: diseño del perfil de mercados meta: Canadá, Reino Unido, Alemania e Italia; la actualización de los objetivos estratégicos; el diseño de las estrategias de producto, precio, distribución y promoción; y un plan de acción en función de las estrategias trazadas, todo lo cual contribuyó al incremento de los ingresos totales del servicio Premium.

PALABRAS CLAVE: Comercialización en hoteles de lujo; Estrategia; Servicio Premium. 


\section{ABSTRACT}

Sales management in a five star hotel is a cornerstone to meet the clients request, mostly in luxury services where the booking and the incomes are key factors that contribute to the development of the hotel. The scientific problem of this research is determined by the insufficient commercialization of the Premium service of Playa Pesquero Resort Suites and SPA. The general objective is the development of a strategy that helps to sell Premium service, which will definitely contribute to the increase of profits and will benefit the booking of the hotel. Several theoretical methods were used for that aim analysis, inductivedeductive, structural-systemic, as well as some empirical: scientific observation, interview, documents revision, and specialist's criteria. The strategy to sell the Premium service consists of four essential components: objectives, phases, tasks and techniques to apply. The partial implementation of this strategy has shown among the most significant results the elaboration of the profile for the target markets: Canada, United Kingdom, Germany and Italy; the definition of the strengths of the product; the formulation of strategies related to price, product, distribution, and promotion through an action plan that illustrates the marked strategies. All this has favored the profits of the hotel Premium service.

KEY WORDS: Commercialization in luxury hotels; Strategy; Premium service.

\section{INTRODUCCIÓN}

En el presente, el turismo en Cuba se abre a lo internacional para brindar un destino que permite enriquecer la vida de clientes mediante experiencias que facilitan el contacto con el clima, la geografía, la realidad social, cultural, el patrimonio y las comunidades. La hotelería cubana está en crecimiento constante y exhibe una capacidad anual que está preparada para recibir unos 5,4 millones de visitantes de todas las latitudes (Marrero, 2017).

En los últimos años se ha incrementado el número de visitantes en un 11,9 por ciento, y en el año 2017 se arribó a la cifra de 4 millones 700 mil visitantes, Cabrisas (2017) y Marrero (2017). Por ello, es importante disponer de una planta hotelera que garantice la calidad establecida en su estándar, y en especial, mejor preparada para recibir al turista de lujo, tan exigente.

Holguín, como uno de los destinos turísticos más importante de Cuba, posee el único servicio Premium marca propia del país, el cual se encuentra en el hotel Playa Pesquero, uno de los más grandes hoteles construidos en Cuba a partir de 2003. Dada la relevancia de continuar el perfeccionamiento de la comercialización del servicio Premium en este hotel, se inició la presente investigación a inicios del 2016 basada en las potencialidades que posee 
por sus atractivos naturales cercanos al mar y la alta calificación de los recursos humanos que en él laboran, sin embargo, presenta las insuficiencias siguientes:

-Limitada toma de decisiones por el Departamento Comercial del hotel en las acciones de promoción y los acuerdos de comarketing, así como escasa participación en ferias de turismo, pues estas se planifican y ejecutan centralmente por la Dirección de Promoción y Ventas del Grupo de Turismo Gaviota S.A.

-Insuficiente publicidad del hotel y del servicio Premium dentro de las redes sociales como: Facebook, Twitter, Google Plus y YouTube y en los sitios de intercambio en Internet, entre los que se destacan: TripAdvisor, Zoover, y TheHolidayCheck, lo que no favorece las ventas a través del sitio web de Gaviota S. A. (www.gaviotahotels.com) y otras plataformas online (IDISO, Booking.com)

-Escasa previsión en la comercialización del hotel en los primeros años de operación, lo que provocó deficiente ocupación y exigió vender a través de precios de oferta upgrade directamente en el hotel, que son inferiores a los precios de contrato.

-Falta de experiencia en la administración de este tipo de servicio que vincula secciones denominadas sólo adultos con hoteles de familia en un mismo resort.

En esta investigación se desarrolla una estrategia para la comercialización del servicio Premium, que contribuya a incrementar los ingresos de este servicio en el hotel Playa Pesquero. La idea a defender es la siguiente: Una estrategia dirigida a la comercialización del servicio Premium del hotel Playa Pesquero, basada en el perfil de los mercados meta, que aporte herramientas para la determinación de las potencialidades del producto para esos mercados y establezca los escenarios y objetivos estratégicos, incrementando los ingresos de este servicio.

\section{DESARROLLO}

\section{Los servicios Premium en los hoteles de lujo}

Antes de abordar las características fundamentales de los servicios Premium resulta necesario realizar un acercamiento a los hoteles de lujo, las cuales son empresas de hospitalidad que tienen una tradición muy antigua arraigada en el viejo continente. Se caracterizan por ofrecer la mejor atención y más amplia gama de servicios, entre ellos: espacio para piscinas, shows y eventos casi todas las noches, salones de gimnasia con profesores y animadores infantiles incluidos, hasta un servicio de guardería para niños. Tienen un espacio para las comidas y veladas con música en vivo, además de una carta desarrollada por varios chefs especializados en la gastronomía de la región Guiñazu (2016), Harmon (2016), Larraiza (2017), Ruz (2017), y Laico (2017). 
Dentro de los servicios de lujos se destacan los Premium. Pérez y Merino (2015) declararon que Premium es un adjetivo que se utiliza para calificar a un servicio o un producto de características especiales, de calidad superior a la media. Lo habitual es que lo Premium sea un privilegio destinado a aquellos consumidores que pagan un monto adicional.

Se trata de un producto elitista, con un elevado nivel de funcionalidad y fiabilidad, gracias a un diseño exquisito; características a las que hay que sumar, además, un precio Premium. Por lo tanto, estos productos están dirigidos publicitariamente a un público objetivo de clase alta, adinerada, de gran poder adquisitivo. Los clientes Premium son compradores racionales que valoran la calidad, funcionalidad, diseño del artículo y, aquí radica la principal diferencia, que tenga una excelente relación calidad-precio, en caso contrario, no efectuarán la compra. Un producto/servicio Premium se encuentra en el extremo de la estrategia de diferenciación, pues ofrece un producto mejor a un precio sustancialmente superior.

Guerrero (2014) detalla siete claves para identificar un producto o servicio Premium, estas son:

1. Es innovador: sus talentos están en la búsqueda constante de la diferenciación. Debido a que son creadores de tendencias, afrontan una insistente percepción de sus competidores.

2. El servicio es limitado: esto ocurre por razones de mercado. El mercado puede ser relativamente reducido o las preferencias de este nicho se diferencian en gran medida del resto.

3. Tiene tradición: El hotel tiene una historia de tradición y búsqueda constante de la calidad. Esta historia es el respaldo en la que los consumidores depositan la confianza. 4. La calidad se antepone al precio.

5. Supera las expectativas de sus clientes: Su consumo provoca sorpresas agradables, un valor extra, un agregado inesperado, un servicio espectacular. De este modo, el proceso de consumo resulta tan agradable como la compra y su recuerdo. El producto se traduce en una vivencia especial y una experiencia inolvidable.

6. Propone o se inserta a una visión del mundo exclusiva y mejorada de la realidad: Lo que se transmuta en un sistema de objetos que llevan este modo de ser a vivencias cotidianas. Se crea una esfera de vida distintiva dentro del mundo cotidiano que es para todos.

7. Es visionaria: Se anticipa al futuro sin dejar de tener en cuenta sus raíces y tradiciones.

Premium refiere a una particularidad de un determinado servicio, aquella que lo hace ser destacado por su extremada calidad y por lo tanto solo asequible por un número reducido de personas. 
En los servicios Premium se siguen las tendencias en tecnología y turismo establecidas por Córdova (2016), De Miguel (2016), y Miralles (2016) estas son: Mercado online, altamente competitivo y complejo; Nuevos modelos de negocios; Big data y marketing. Significa esto que las tendencias que siguen los clientes de lujo están relacionadas con las tendencias globales de las tecnologías de la información y las comunicaciones, en las que se realza la importancia del flujo de información promocional y la innovación en el marketing online.

\section{Metodologías y procedimientos para formular estrategias comerciales}

Existen diversas propuestas a nivel teórico para la confección de planes de comercialización, utilizadas indistintamente de acuerdo con la complejidad del estudio y de la organización en que se aplique (Figueiras, 2018). En el contexto cubano se ejecuta el modelo de dirección estratégica que contribuye a lograr la integración de los niveles estratégico, táctico y operativo en las organizaciones con vista a elevar el desempeño de éstas. Al respecto, se destacan resultados de investigaciones entre las que se encuentran:

-Palao (2001): Procedimiento para la elaboración de un plan de marketing de un destino turístico.

-Peña (2006): Procedimiento para posicionar el destino Holguín en los mercados emisores que lo visitan.

-Cardet (2007): Modelo sobre diseño de estrategias en el área de ciencia e innovación tecnológica.

-Rivero (2008): Procedimiento para la gestión comercial de un destino turístico.

-Rodríguez (2008): Metodología para la planificación estratégica en el área comercial.

-Hernández (2010): Procedimiento para la elaboración e implementación del plan de marketing en pequeños y medianos hoteles de tránsito del destino Villa Clara.

-Escalona (2010): Procedimiento para la formulación de estrategias de un mercado emisor de un destino.

-Medina (2012): Procedimiento para la formulación de estrategias comerciales de un destino turístico para un mercado emisor.

Las propuestas anteriores tienen en común el cumplimiento de varios de los aspectos básicos del proceso de planificación estratégica, estos son: el diagnóstico interno y externo, la definición de la misión y la visión, el empleo de la matriz Debilidades - AmenazasFortalezas - Oportunidades (DAFO), la determinación de los objetivos estratégicos y la formulación e implementación de estrategias. De ellas, se considera que el procedimiento aportado por Medina (2012) recoge la mejor experiencia precedente de acuerdo con el objetivo de la presente investigación, e incorpora un elemento de vital importancia: la 
definición del perfil de los mercados meta, en el que se abarcan los elementos que aseguran una amplia caracterización de los mismos, aspecto que se considera indispensable para la efectiva formulación de estrategias comerciales en función de satisfacer esos mercados.

Al considerar que la presente investigación concibió como campo práctico la comercialización del servicio Premium en el hotel Playa Pesquero, fue necesario analizar a profundidad el alcance de algunos pasos y técnicas propuestos por Medina (2012), debido a que no se ajustan específicamente a las características de un servicio tan peculiar como el Premium y con diversos mercados meta.

A modo de síntesis, se considera que el desarrollo de estrategias comerciales es necesario concebirlo en fases con el mismo nivel de importancia, que van desde: la fase del análisis estratégico, la cual debe incluir la selección de un equipo de especialistas competente, la elaboración del perfil de los mercados meta y la determinación de las potencialidades del producto para esos mercados, también es relevante la determinación de los escenarios y de los factores clave del éxito; la fase de formulación estratégica que debe incluir la determinación de las proyecciones de arribo e ingresos, los objetivos estratégicos, la formulación de las estrategias precio, producto, distribución y promoción y la evaluación de los factores que limitan la implementación de esas estrategias; y por último, la fase de evaluación estratégica, que debe contener el control de las estrategias, la evaluación de indicadores establecidos y la comunicación de los resultados alcanzados. Todo lo anterior contribuye a una mejor concepción de la comercialización del servicio Premium en hoteles de lujo.

\section{Estrategia para la comercialización del servicio Premium en el hotel Playa Pesquero}

El objetivo general de la estrategia es favorecer la comercialización del servicio Premium del hotel Playa Pesquero, basada en: la confección del perfil de los mercados metas, determinación de las potencialidades del producto para dichos mercados, así como en la determinación de los escenarios y objetivos estratégicos para el diseño de las estrategias de producto, precio, distribución y promoción, y su control, de manera que se incrementen los ingresos en este servicio.

La estrategia que se propone, se estructura en cuatro componentes esenciales por los que transcurre el proceso de comercialización del servicio Premium del hotel Playa Pesquero, estos son: objetivos, fases, tareas, y técnicas a emplear. La representación de la estructura de análisis a seguir para la comercialización del servicio Premium se muestra en la Figura $\mathrm{N}^{\circ} 1$. 


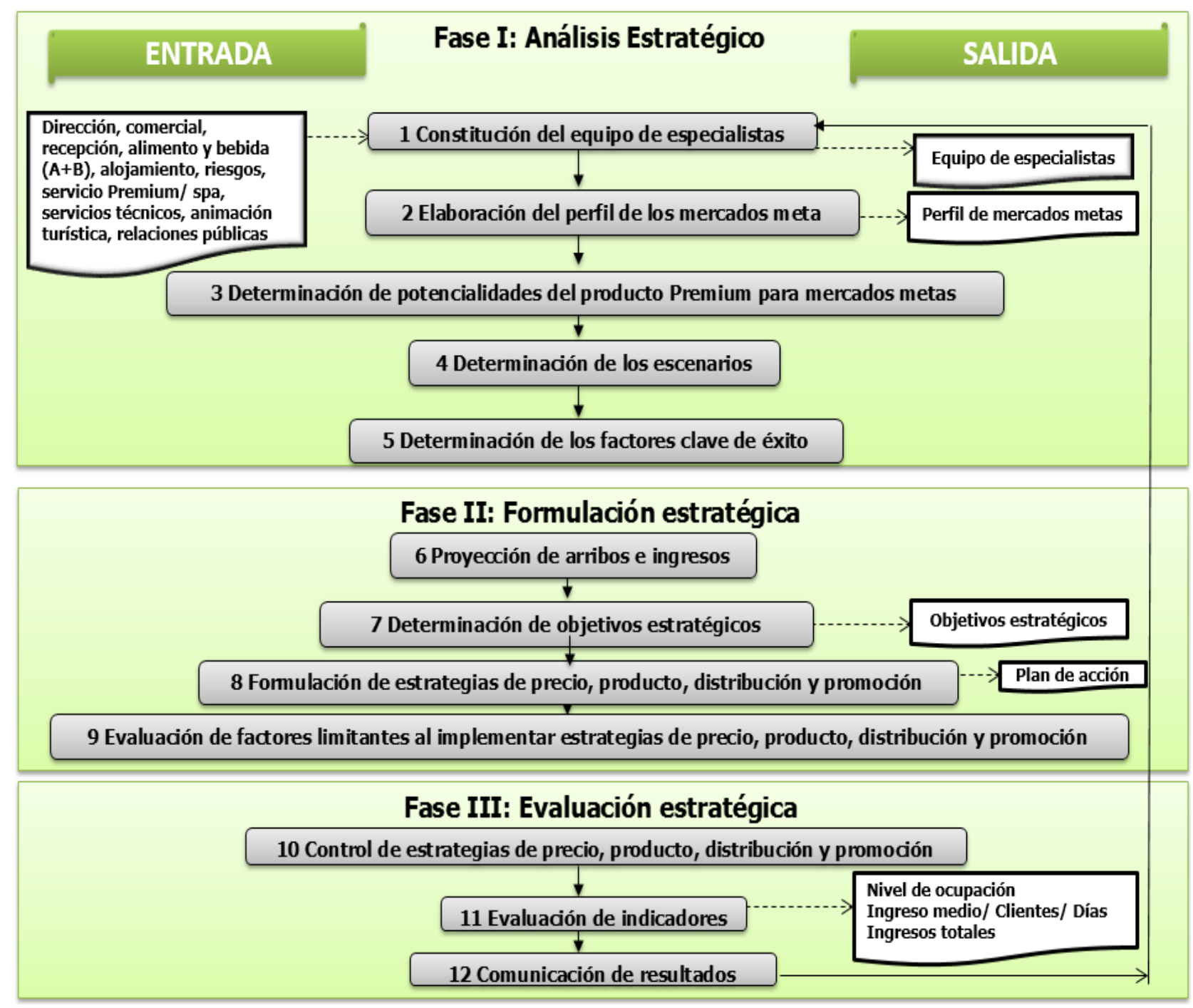

Figura $N^{\circ}$ 1. Representación gráfica de la estructura a seguir para la comercialización del servicio Premium del hotel Playa Pesquero

Fuente: Elaboración Propia

Las fases de la estrategia se describen a continuación:

Fase I. Análisis estratégico

Tarea 1. Constitución del equipo de especialistas

Se conformó un equipo de diez especialistas, representativo de los cargos relacionados con el servicio Premium (dirección, comercial, recepción, alimento y bebida $(A+B)$, alojamiento, riesgos, servicio Premium/spa, servicios técnicos, animación turística y relaciones públicas). En esta composición se encuentran: dos másteres en gestión turística con experiencia en la operación hotelera, tres especialistas comerciales con más de ocho años de experiencia, un directivo del servicio Premium, dos directivos del hotel, un especialista de área de reserva y otro del área de servicio gastronómico. Se seleccionó como responsable del equipo al especialista comercial. Fueron impartidas por el especialista en riesgos dos capacitaciones sobre las tendencias en la comercialización de hoteles de

\footnotetext{
Visión de Futuro" Año 16, Volumen N²3 N², Julio - Diciembre 2019 - Pág. 252 - 273

URL de la Revista: http://visiondefuturo.fce.unam.edu.ar/index.php/visiondefuturo/index

URL del Documento: http://visiondefuturo.fce.unam.edu.ar/index.php/visiondefuturo/issue/view/16

ISSN 1668 - 8708 - Versión en Línea

E-mail: revistacientifica@fce.unam.edu.ar
} 
lujo, las que fueron desarrolladas en el hotel en el primer semestre de 2017 y resultaron de gran utilidad para analizar las peculiaridades del servicio Premium, sus potencialidades y retos.

Tarea 2. Elaboración del perfil de los mercados meta

Para elaborar el perfil de los mercados meta se debe tener en cuenta la importancia del estudio de las variables de los perfiles turísticos establecidos por Medina (2012), Beauchamp y Barnes (2015) y De la Mora (2017). Según la política comercial del Grupo Gaviota S.A., se destacan como los principales mercados meta en Cuba a: Canadá, Reino Unido, Alemania e Italia. Una síntesis del perfil de Canadá se muestra a continuación:

-Comportamiento del consumidor: Actualmente se interesa más en la calidad del producto, su origen, composición y precio, especialmente desde la crisis financiera mundial de 2009. También es excesivamente sensible a la publicidad y tiende a comprar productos de moda. En contraste con los últimos años, la confianza de los consumidores se ha reforzado gracias al repunte de la economía. Los consumidores canadienses exigen un servicio de venta y posventa de alta calidad. Es sumamente útil proponer una atención telefónica gratuita para mantener el contacto con los consumidores. Los consumidores canadienses son cada vez más sensibles a los problemas relacionados con el medio ambiente y el excesivo consumo, y recurren cada vez más a Internet para sus compras: en la última década las ventas por Internet han crecido a un ritmo mayor que las ventas en comercios tradicionales (el gasto per cápita en compras por Internet aumentó 17\% únicamente entre 2015 y 2016, con una cifra de 661 dólares canadienses (CAD)). Los consumidores más jóvenes lideran el crecimiento de la compra en línea a través del uso creciente de los Smartphone y aparatos móviles al comprar en Internet. El valor elevado de la vivienda ha alejado a los consumidores más jóvenes de la compra de una propiedad, lo que ha generado una demanda más lenta de diferentes tipos de productos para el hogar.

-Perfil del consumidor y su poder adquisitivo: El nivel de vida en Canadá es uno de los más altos del mundo. Los consumidores confían en que el empleo seguirá pleno, esto estimula el consumo, al menos en algunas provincias. Se observa un cambio en las tendencias de compra, sobre todo entre los jóvenes canadienses. Los principales objetivos de la mayoría de jóvenes son terminar sus estudios, comprar una vivienda y tener hijos. Los adultos de mediana edad y los de la generación del baby-boom (1946-1966) dedican mucho tiempo al ocio. Los canadienses se preocupan por su alimentación y no dudan en comprar productos saludables, naturales y biológicos. Le dan mucha importancia a todo lo relacionado con el confort y el bienestar. Las personas de 60 años o más constituyen el segmento de consumidores que crece más rápido. La población de Canadá es una de la más diversa entre los países occidentales modernos, y esta tendencia debe prolongarse en los años venideros con la llegada de más inmigrantes. Cada vez más, las olas de 
inmigrantes influencian el perfil del consumidor canadiense y los hábitos de compra, lo que modifica las compras de comestibles en particular.

-Recurso al crédito por parte del consumidor: En Canadá, el uso del crédito por los consumidores es frecuente. Entre 2012 y 2017, el crédito de consumo aumentó anualmente un 2,5\%, lo que representó un total de 571,1 mil millones de dólares canadienses (CAD). Se emplea para comprar a crédito bienes y servicios y para financiar compras ya realizadas. El crédito más corriente es el préstamo de liquidez para financiar compras con plazos (generalmente artículos grandes como muebles o automóviles, pero también para pagar la gasolina y otros bienes perecederos). De forma similar, la deuda doméstica acumulada, hipotecas, préstamos de garantías y otras formas de crédito de consumo no personal, ha aumentado con una tasa anual de $1,8 \%$ en este período de cinco años. El endeudamiento ha crecido con un índice más elevado que la población, esto causa un aumento radical de los niveles de deuda por habitante.

-Medios publicitarios:

- Televisión: La publicidad televisada se concentra principalmente en el grupo de población de 35 a 49 años. Mucho más efectiva que el uso aislado de la televisión es la combinación de televisión, publicidad impresa e Internet, que aumenta la intención de compra más del $47 \%$.

- Prensa: La publicidad impresa aún ejerce un fuerte impacto entre los canadienses, a pesar de su disminución en los últimos años, para dar paso a la publicidad on-line. Sin embargo, el costo de los medios tradicionales de publicidad masiva, como los periódicos, es muy elevado. La industria de periódicos muestra un declive a nivel del gasto, de 5,5\% en 2017 y $3 \%$ en 2018. Por ello, los medios planifican estrategias para desplazarse hacia soportes digitales más que impresos. Los periódicos en 2017 representaron el 17\% del gasto total en comparación con 43\% para el soporte digital.

- Correo: En Canadá se conoce este tipo de publicidad como Courrier Poubelle o Junk mail (correo basura). Los folletos publicitarios que más se conservan y aprecian son los semanarios de alimentación y de productos farmacéuticos. Cuando los niños vuelven a la escuela las familias prestan especial atención a los folletos publicitarios de ropa y material escolar.

- Medios de transporte: Desde hace muchos años la publicidad invade el transporte público, las estaciones de trenes y las paradas de autobuses. La venta de publicidad en exterior creció un $3 \%$ en 2017 , en gran parte gracias a un aumento del $13 \%$ de la publicidad digital en exterior en el año.

- Radio: La publicidad radiofónica se escucha sobre todo en los coches, oficinas y comercios. La publicidad tradicional masiva en Canadá, como la radio, tiene un costo 
elevado. La publicidad radial es mayoritariamente local. En 2016-2017, las ventas de publicidad en radios locales confrontaron el declive más pronunciado en cinco años. Por otra parte, las ventas a nivel nacional crecieron un 1,2\% en comparación con el año anterior.

- Web: Cerca de 22 millones de canadienses utilizan Internet mensualmente. Esta red se ha convertido en un medio irreemplazable de comunicación, contacto, búsqueda y compra para consumidores y empresas por igual. La publicidad on-line ejerce un impacto mayor que la televisión en la intención de compra de los canadienses. Los publicistas estudian a los consumidores e invierten una parte cada vez mayor de su presupuesto publicitario en los medios de comunicación interactivos. La publicidad online se centra sobre todo en la población de 18 a 34 años. Un factor crucial para promover productos y servicios es la creación y mantenimiento de una página web profesional. Se previó que la venta de publicidad en medios sociales aumentara en $35 \%$, la venta de publicidad en videos $20 \%$, y la venta de publicidad en búsquedas $13 \%$. En su conjunto, se proyectó que la venta de publicidad digital aumentará un 16\% en 2017, con una cifra de 7 mil millones de dólares canadiense (CAD). Los hábitos de compra de los consumidores canadienses además son influenciados por los medios sociales y un número creciente de ellos admite que es influenciado por la lectura de reseñas, comentarios y reacciones en plataformas de medios sociales, junto con anuncios en línea. Mientras que los distribuidores locales tradicionalmente van a la zaga de los distribuidores estadounidenses a nivel de su presencia en Internet, se proyecta que en los próximos años el crecimiento de las compras en línea sea animado principalmente por la mayor presencia en Internet de estos mismos distribuidores locales.

Tarea 3. Determinación de potencialidades del producto Premium para mercados meta

A través de una dinámica grupal el equipo de especialistas realizó el análisis de la Matriz DAFO, la que se muestra en la tabla siguiente:

Tabla №1. Análisis DAFO

\begin{tabular}{|c|c|}
\hline Oportunidades & Amenazas \\
\hline 01. Discreta emisión de visitantes desde & A1. Fluctuaciones en las tasas de cambio. \\
\hline $\begin{array}{l}\text { Estados Unidos hacia Cuba y hacia el destino } \\
\text { Holguín. }\end{array}$ & $\begin{array}{l}\text { A2. Incremento de la competencia en el área con } \\
\text { la apertura del nuevo hotel lberostar. }\end{array}$ \\
\hline $\begin{array}{l}\text { O2. Creciente importancia otorgada a la calidad } \\
\text { de los servicios por los clientes. }\end{array}$ & $\begin{array}{l}\text { A3. Presencia de la planta real del hotel Río de } \\
\text { Oro en el destino. }\end{array}$ \\
\hline $\begin{array}{l}\text { O3. Entrada de nuevas aerolíneas en el destino, } \\
\text { como America Air line y Copa Air line, que }\end{array}$ & $\begin{array}{l}\text { A4. Incremento de la competencia en el Caribe } \\
\text { con altos estándares de calidad y menores }\end{array}$ \\
\hline
\end{tabular}




\begin{tabular}{|c|c|}
\hline $\begin{array}{l}\text { operan con segmentos de mercado de lujo. } \\
\text { O4. Incremento de un vuelo de Thomas Cook. } \\
\text { O5. Potenciación en el destino de la } \\
\text { diversificación de mercados como: Cono Sur, } \\
\text { Polonia, y Rusia. } \\
\text { 06. El servicio Premium es una sección } \\
\text { independiente dentro del hotel Playa Pesquero. }\end{array}$ & $\begin{array}{l}\text { precios. } \\
\text { A5. Marcada estacionalidad de la ocupación. } \\
\text { A6. Irregularidad en los suministros. } \\
\text { A7. Poca afluencia al destino de vuelos con } \\
\text { primera clase. }\end{array}$ \\
\hline $\begin{array}{l}\text { Fortalezas } \\
\text { F1. Único hotel cinco estrellas marca propia en } \\
\text { Cuba con un servicio Premium. } \\
\text { F2. Acceso directo a la playa. } \\
\text { F3. Cortesía, amabilidad y preparación del } \\
\text { personal. } \\
\text { F4. Accesibilidad para minusválidos. } \\
\text { F5. Servicio de SPA. } \\
\text { F6. Variedad de bebidas nacionales e } \\
\text { internacionales incluidas. } \\
\text { F7. Lobby Bar con servicio } 24 \text { horas. } \\
\text { F8. Servicio de Wireless Fidelity (Wi-Fi) incluido. }\end{array}$ & $\begin{array}{l}\text { Debilidades } \\
\text { D1. Insuficiente promoción del servicio Premium } \\
\text { en los principales emisores de clientes como } \\
\text { Canadá, Reino Unido, Alemania e Italia. } \\
\text { D2. Áreas abiertas vulnerables a la lluvia. } \\
\text { D3. Las habitaciones no tienen vista al mar. } \\
\text { D4. Faltan baños y duchas en la playa. } \\
\text { D5. Precio alto para un producto pionero. } \\
\text { D6. Insuficiente animación nocturna. } \\
\text { D7 Falta de boutiques para compras de los } \\
\text { clientes de lujo. }\end{array}$ \\
\hline
\end{tabular}

Fuente: Elaboración Propia

Evaluación de los factores externos e internos: Para la evaluación de los factores se emplearon de forma respectiva la Matriz de Evaluación de Factores Internos (MEFI) y la Matriz de Evaluación de Factores Externos (MEFE). El resultado que se obtuvo al realizar la MEFI es de 2,23 por debajo de 2,50, lo que indica que en el servicio Premium predominan las debilidades sobre las fortalezas, por lo que se encuentra en una posición desventajosa. En el caso de la MEFE se obtuvo un valor de 2,58 superior a los 2,50, por lo tanto predominan las oportunidades sobre las amenazas, lo que se considera una ventaja para el servicio Premium del hotel.

Análisis de la Matriz DAFO: Para realizar la matriz DAFO se redujeron los factores externos e internos al dejar solamente los de mayor importancia. Fue utilizado para ello el criterio de colocar por la parte superior las oportunidades y las amenazas que hayan obtenido 4 y 1 punto respectivamente y por la izquierda hacia abajo las fortalezas y debilidades con 4 y 1 respectivamente. Una vez realizada la matriz se concluyó que el servicio Premium, se encuentra en el cuadrante III (Debilidades-Oportunidades), por lo que se deben aprovechar las oportunidades para minimizar las amenazas existentes en el entorno.

El análisis de la matriz DAFO y de los factores internos y externos fueron presentados 
al consejo de dirección para la toma de decisiones. Luego el equipo de especialistas procedió a determinar las potencialidades del servicio Premium para los mercados metas, estas son:

- Naturales: El servicio Premium se localiza en el litoral norte, en la playa Pesquero, en un rico entorno natural dentro del parque Cristóbal Colón y en el perímetro de uno de los corredores de aves migratorias más importantes de la isla.

- Infraestructuras y servicios disponibles: El servicio Premium cuenta con 56 habitaciones de un área de 93,68 $\mathrm{m}^{2}$ con una recepción independiente, lobby bar, sala de lectura, restaurante gourmet para desayuno, almuerzo y cena, siempre a la carta, tiene además snack bar, piscina, solárium, servicio de mayordomo y pool concierge, room service incluido, con menú incluido las 24 horas, Wi-Fi, carta de almohadas, 20\% de descuento sobre los servicios de lavandería, 15\% de descuento sobre los tratamientos del spa, late check out gratis según disponibilidad, amenities superiores en el baño y acceso a todas las facilidades y reservas de los restaurantes del hotel Playa Pesquero.

- Culturales: Otro atractivo es sin dudas el interés histórico de la zona, particularmente Cayo Bariay, donde tuvo lugar el primer encuentro entre la civilización europea y la aborigen; el museo indocubano Chorro de Maíta en el municipio Banes, que ofrece la muestra más importante de la cultura precolombina en Las Antillas, como testigo principal de la convivencia de ambas culturas, y la ciudad de Holguín con viarios sitios históricos de relevancia como la Loma de la Cruz, el museo La Periquera, entre otros.

- Oferta: Los deportes náuticos desarrollados son el kayak, bicicleta acuática y el catamarán; posee otras ofertas como: el baño con delfines, el día campestre, la súper campiña, Santiago de Cuba en bus, la pesca a vara, la pesca a cordel, el crucero del sol, entre otras.

- Formación: Cuenta con 73 trabajadores (25 en servicio, 17 en alojamiento, 13 en área de cocina, 7 en recepción, 6 mayordomos, 2 en higiene, 2 en dirección y 1 en servicio técnicos). En las áreas de recepción, servicio, y mayordomo los trabajadores dominan más de dos idiomas. Los de las áreas de alojamiento y cocina son graduados de la escuela de hotelería y turismo con resultados relevantes, además de contar con la asesoría de un chef extranjero.

Tarea 4. Determinación de los escenarios

Como resultado de la aplicación del Método de construcción de escenarios de Vieytes (2010) se determinaron tres escenarios, de ellos fue seleccionado el escenario medio: Caracterizado por el incremento de los vuelos regulares y chárter al destino Holguín, por la flexibilización del bloqueo económico a Cuba por parte de Estados Unidos, por la estabilidad económica internacional, por un incremento de la comunicación promocional del servicio "Visión de Futuro" Año 16, Volumen N²3 N², Julio - Diciembre 2019 - Pág. 252 - 273 
Premium del hotel Playa Pesquero, por una adecuada disponibilidad de tecnología de las comunicaciones y la información en el producto, por el incremento de la competencia en Cuba y en el Caribe, y una alta percepción por parte de los clientes de la calidad del servicio que reciben en la instalación.

Tarea 5. Determinación de los factores clave de éxito

Para determinar los factores claves de éxito (FCE) se consideró las preferencias de los clientes Premium descritas en el perfil y se realizó una dinámica grupal para determinar cuáles de los elementos definidos en el perfil son más influyentes en el incremento de los arribos al servicio Premium, definiéndose los siguientes:

- FCE 1: Acceso a la información de los atributos del servicio Premium por parte de los turoperadores y clientes finales.

- FCE 2: Exclusividad y calidad del servicio de las ofertas del servicio Premium donde obtengan en cada momento una experiencia única.

- FCE 3: Posibilidad de ofertas exclusivas.

Fase II. Formulación estratégica

Tarea 6. Proyección de arribos e ingresos

Para realizar la proyección de los Clientes/ Días y los Ingresos en Pesos Cubanos Convertibles (CUC) hasta el 2021 se valoró la operación del servicio Premium desde 2015 a 2017. Este análisis permitió realizar la proyección, la que puede observarse en la tabla siguiente.

Tabla N². Proyección de Clientes/Días e Ingreso hasta el 2021

\begin{tabular}{|l|c|c|c|c|}
\hline \multicolumn{1}{|c|}{ Año } & 2018 & 2019 & 2020 & 2021 \\
\hline Clientes/Días & 15000 & 17000 & 19000 & 21000,00 \\
\hline Ingreso (CUC) & 1350,00 & 1615,00 & 1900,00 & 2205,00 \\
\hline
\end{tabular}

Fuente: Elaboración Propia

Tarea 7. Determinación de objetivos estratégicos

Para determinar los objetivos se comenzó por proyectar las variables estratégicas de marketing (segmentación, mercado meta y posicionamiento) para el segmento de mercado en estudio, después se determinaron los objetivos estratégicos para cada uno de los factores claves de éxito con un horizonte temporal hasta el 2021. A través de la dinámica grupal y el consenso del equipo, las variables estratégicas quedaron definidas de la forma siguiente:

-Segmentación: Se tomó como criterios para la segmentación variables geográficas, económicas y de comportamiento organizacional.

-Mercado meta: Turistas con intereses vacacionales en hoteles de playa, de alto poder

Visión de Futuro" Año 16, Volumen N²3 N², Julio - Diciembre 2019 - Pág. 252 - 273

URL de la Revista: http://visiondefuturo.fce.unam.edu.ar/index.php/visiondefuturo/index

URL del Documento: http://visiondefuturo.fce.unam.edu.ar/index.php/visiondefuturo/issue/view/16

ISSN 1668 - 8708 - Versión en Línea

E-mail: revistacientifica@fce.unam.edu.ar 
adquisitivo, procedentes de Canadá, Reino Unido, Alemania e Italia.

Para estos segmentos se propone que el servicio Premium trabaje en el posicionamiento siguiente: Servicio Premium del hotel Playa Pesquero caracterizado por el reconocimiento a la excelencia hotelera en el Caribe y altos niveles de satisfacción de los clientes. Luego se procedió a definir los objetivos estratégicos, estos son:

-Objetivo No. 1: Alcanzar una ocupación promedio anual del $70 \%$ en el servicio Premium para el 2021. Los criterios de medida de este objetivo por años se muestran en la Fig. $\mathrm{N}^{0} 2$.



Figura No2. Proyección de la ocupación promedio hasta 2021 Fuente: Elaboración Propia

-Objetivo N²: Alcanzar un Ingreso medio/Clientes/ Días de105,00 CUC en 2021. Los criterios de medida de este objetivo por años se muestran en la Fig. № 3.

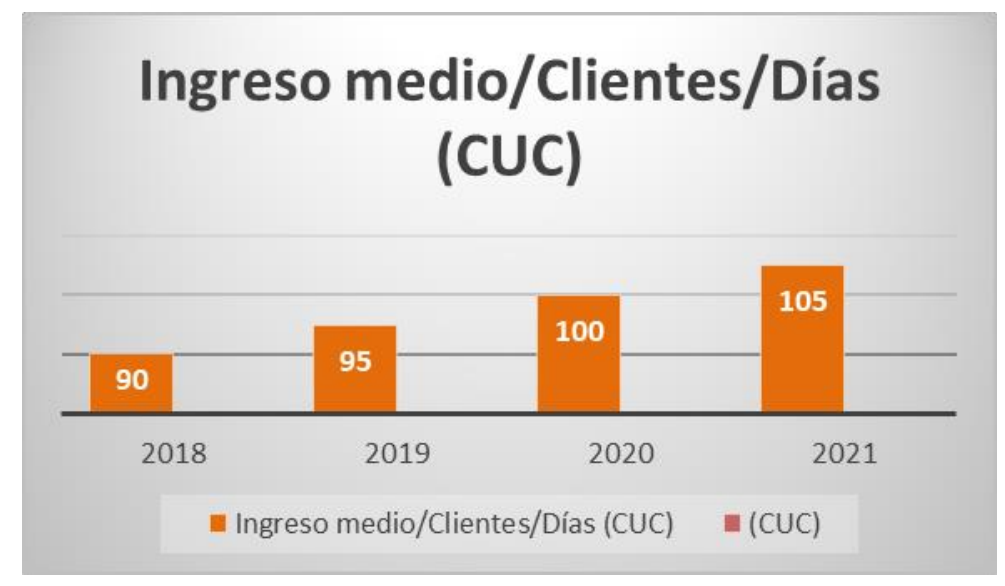

Figura N³. Proyección del Ingreso medio/ Clientes/Días hasta 2021 Fuente: Elaboración Propia

Tarea 8. Formulación de estrategias de precio, producto, distribución y promoción

Para cumplir los objetivos planteados anteriormente se propuso, a través del consenso del equipo de especialistas, un conjunto de líneas estratégicas, estas son: 
1. Agregar valores al producto en correspondencia con su estándar de calidad.

2. Establecer y vender todo el año el servicio Premium con los precios de contrato con los turoperadores.

3. Diversificar los canales de distribución del producto.

4. Incrementar la comunicación promocional del producto en los mercados meta.

5. Captar nuevos segmentos de clientes procedentes de los mercados potenciales: Rusia, Polonia, Austria, Chile y Estados Unidos, así como de segmentos de clientes de negocios, eventos y diplomáticos.

Para cumplir los objetivos propuestos y en correspondencia con las líneas estratégicas, se diseñó un plan de acción con 18 acciones que abarca las variables operativas del marketing (producto, precio, distribución, promoción) para el periodo 2017/ 2019.

Tabla N03. Plan de acción para la comercialización del servicio Premium del hotel Playa Pesquero

\begin{tabular}{|c|c|c|c|}
\hline Línea estratégica & Acciones & Responsable & $\begin{array}{l}\text { Fecha } \\
\text { cumplimiento }\end{array}$ \\
\hline \multirow{3}{*}{$\begin{array}{lr}\text { Agregar valor al } \\
\text { producto } & \text { en } \\
\text { correspondencia con } \\
\text { su estándar de } \\
\text { calidad }\end{array}$} & $\begin{array}{l}\text { Capacitar a los trabajadores sobre las } \\
\text { tendencias actuales de su especialidad } \\
\text { en los servicios Premium }\end{array}$ & $\begin{array}{l}\text { Jefe } \\
\text { Recursos } \\
\text { Humanos, Jefe } \\
\text { servicio Premium }\end{array}$ & $\begin{array}{l}\text { I Semestre } \\
2018\end{array}$ \\
\hline & $\begin{array}{l}\text { Garantizar nuevos proveedores que } \\
\text { aseguren los suministros indispensables } \\
\text { para garantizar la calidad de este tipo de } \\
\text { servicio }\end{array}$ & Jefe de Compras & $\begin{array}{l}\text { II Semestre } \\
2018\end{array}$ \\
\hline & $\begin{array}{l}\text { Incorporar al producto el servicio de } \\
\text { tiendas boutique }\end{array}$ & $\begin{array}{l}\text { Jefe servicio } \\
\text { Premium }\end{array}$ & Enero 2019 \\
\hline \multirow{3}{*}{$\begin{array}{l}\text { Establecer y vender } \\
\text { todo el año el } \\
\text { servicio Premium con } \\
\text { los precios de } \\
\text { contrato con los } \\
\text { turoperadores }\end{array}$} & $\begin{array}{l}\text { Confeccionar ficha de costo promedio por } \\
\text { cliente del servicio Premium }\end{array}$ & $\begin{array}{l}\text { Jefe Comercial, } \\
\text { Jefe Económico }\end{array}$ & Julio 2018 \\
\hline & $\begin{array}{l}\text { Realizar estudios de precio de la } \\
\text { competencia }\end{array}$ & Jefe Comercial & $\begin{array}{l}\text { II Semestre } \\
\text { de } 2018\end{array}$ \\
\hline & $\begin{array}{l}\text { Estudiar y presentar al Grupo Gaviota S.A } \\
\text { propuestas de precios estacionarios y } \\
\text { promocionales de acuerdo a las } \\
\text { temporadas }\end{array}$ & Jefe Comercial & Mayo 2018 \\
\hline \multirow{4}{*}{$\begin{array}{l}\text { Diversificar } \\
\text { canales } \\
\text { distribución } \\
\text { producto }\end{array}$} & $\begin{array}{l}\text { Crear alianzas estratégicas con nuevos } \\
\text { turoperadores y agencias de viaje que } \\
\text { vendan paquetes a clientes de lujo como: } \\
\text { Pegas Touristik, CIC Colombia, } \\
\text { Havanatur Chile y Argentina }\end{array}$ & Jefe Comercial & Mayo 2018 \\
\hline & $\begin{array}{l}\text { Identificar las líneas aéreas que conectan } \\
\text { los mercados meta con el destino Holguín }\end{array}$ & Jefe Comercial & Abril 2018 \\
\hline & $\begin{array}{l}\text { Ampliar las facilidades para la venta por } \\
\text { Internet del producto Premium en el sitio } \\
\text { web del hotel }\end{array}$ & $\begin{array}{l}\text { Jefe Comercial, } \\
\text { Jefe Economía }\end{array}$ & $\begin{array}{l}\text { II Semestre } \\
2018\end{array}$ \\
\hline & $\begin{array}{l}\text { Crear y aplicar mecanismos que } \\
\text { estimulen a los turoperadores a } \\
\text { incrementar las ventas del servicio }\end{array}$ & Jefe Comercial & $\begin{array}{l}\text { II Semestre } \\
2018\end{array}$ \\
\hline
\end{tabular}

Visión de Futuro" Año 16, Volumen N²3 N², Julio - Diciembre 2019 - Pág. 252 - 273

URL de la Revista: http://visiondefuturo.fce.unam.edu.ar/index.php/visiondefuturo/index

URL del Documento: http://visiondefuturo.fce.unam.edu.ar/index.php/visiondefuturo/issue/view/16 


\begin{tabular}{|c|c|c|c|}
\hline Línea estratégica & Acciones & Responsable & $\begin{array}{l}\text { Fecha } \\
\text { cumplimiento }\end{array}$ \\
\hline & Premium & & \\
\hline \multirow{4}{*}{$\begin{array}{ll}\text { Incrementar } & \text { la } \\
\text { comunicación } & \\
\text { promocional } & \text { del } \\
\text { producto en } & \text { los } \\
\text { mercados meta } & \end{array}$} & $\begin{array}{l}\text { Diseñar un catálogo específico del } \\
\text { servicio Premium para la red de ventas }\end{array}$ & Jefe Comercial & $\begin{array}{l}\text { II Semestre } \\
2017\end{array}$ \\
\hline & $\begin{array}{l}\text { Actualizar los atractivos y ofertas del } \\
\text { servicio Premium y del entorno en el sitio } \\
\text { web del hotel mediante la interacción con } \\
\text { los clientes }\end{array}$ & Jefe Comercial & $\begin{array}{l}\text { I Semestre } \\
2018\end{array}$ \\
\hline & $\begin{array}{l}\text { Auditar las acciones de co-marketing en } \\
\text { busca de mayor efectividad en las } \\
\text { acciones de comunicación promocional }\end{array}$ & Jefe Comercial & $\begin{array}{l}\text { II Semestre } \\
2018\end{array}$ \\
\hline & $\begin{array}{l}\text { Gestionar la reputación online del servicio } \\
\text { Premium en el sitio web del hotel, en el } \\
\text { sitio web de Gaviota S. A. y en las redes } \\
\text { sociales (Tripadvisor, Zoover, Monarc, } \\
\text { Holidaycheck) }\end{array}$ & Jefe Comercial & $\begin{array}{l}\text { I Semestre } \\
2018\end{array}$ \\
\hline \multirow{4}{*}{$\begin{array}{lr}\text { Captar } & \text { nuevos } \\
\text { segmentos } & \text { de } \\
\text { clientes procedentes } \\
\text { de los mercados } \\
\text { potenciales: Rusia, } \\
\text { Polonia, } & \text { Austria, } \\
\text { Chile y } & \text { Estados } \\
\text { Unidos, así como de } \\
\text { segmentos } \\
\text { clientes de negocios, } \\
\text { eventos } \\
\text { diplomáticos }\end{array}$} & $\begin{array}{l}\text { Realizar acciones de comunicación } \\
\text { promocional dirigidas a los segmentos } \\
\text { Rusia, Polonia, Austria, Chile y Estados } \\
\text { Unidos en los medios donde los mismos } \\
\text { se informan }\end{array}$ & Jefe Comercial & $\begin{array}{l}\text { II Semestre } \\
2018\end{array}$ \\
\hline & $\begin{array}{l}\text { Ampliar las negociaciones a través del } \\
\text { MINTUR con los turoperadores rusos }\end{array}$ & Jefe Comercial & $\begin{array}{l}\text { I Semestre } \\
2018\end{array}$ \\
\hline & $\begin{array}{l}\text { Identificar la red de ventas para clientes } \\
\text { de lujo en estos segmentos }\end{array}$ & Jefe Comercial & $\begin{array}{l}\text { II Semestre } \\
2018\end{array}$ \\
\hline & $\begin{array}{l}\text { Negociar los eventos con las entidades } \\
\text { Suchel S. A., Cervecería Bucanero S. A., } \\
\text { Empresa de Telecomunicaciones } \\
\text { (ETECSA), entre otras }\end{array}$ & Jefe Comercial & $\begin{array}{l}\text { II Semestre } \\
2018\end{array}$ \\
\hline
\end{tabular}

Fuente: Elaboración Propia

Tarea 9. Evaluación de factores limitantes al implementar estrategias de precio, producto, distribución y promoción

Para evaluar las posibilidades reales de implementar las estrategias, se realizó el diagnóstico de los factores que podían afectar de manera negativa a las mismas, para lo cual se utilizó el modelo de las Siete Eses de Mckinsey, descritas por Stoner, Freeman y Gilbert (1996), cuyos resultados fueron:

- Estructura: Esta permite desarrollar las estrategias pues el servicio Premium es un área dentro del hotel que intercambia con el resto de los servicios generales.

- Sistema: La capacitación y los sistemas contables en el hotel favorecen el adecuado funcionamiento de los sistemas de gestión en el servicio.

- Estilo: La Dirección Nacional del Grupo de Turismo Gaviota S.A. y la dirección del hotel están involucradas en la obtención de resultados superiores, y dan prioridad a las estrategias y sus acciones.

- Integración del personal: El Consejo de Dirección del hotel valora la contribución del staff al cumplimiento de los objetivos del servicio Premium, y en consecuencia, procura 
una adecuada selección y capacitación, tanto de los directivos como de los trabajadores del servicio en general.

- Habilidades: El servicio Premium se destaca y es reconocido por la organización de eventos y cenas, distinguiéndose por la calidad en los servicios, por ofertar un ambiente acogedor, donde el cliente puede apreciar de forma muy positiva su estancia.

- Metas de orden superior: Entre ellas se trabaja en el logro de ser uno de los principales destinos turísticos de Cuba y del Caribe, que se diferencia por la peculiar combinación de sus atractivos naturales, culturales, con una oferta diversificada, que permite satisfacer a sus clientes, al brindar y desarrollar un producto sostenible.

- Estrategias: Los directivos del servicio Premium tienen bien definidos los objetivos estratégicos, los que están atentos al cumplimiento de las metas trazadas a corto, mediano y largo plazo.

De acuerdo con el análisis anterior y a modo de resumen, las estrategias se pueden aplicar hasta 2021 por su carácter flexible, y además, por la preparación del equipo de trabajo encargado de su aplicación, el cual posee competencias para enfrentar alguna limitación, tanto interna como externa, que pueda surgir e incidir en el cumplimiento de los objetivos trazados.

Fase III. Evaluación estratégica

Tarea 10. Control de estrategias de precio, producto, distribución y promoción

En total fueron propuestas 18 acciones, de ellas fueron cumplidas 8, para un $44,44 \%$, estas son:

1. Capacitar a los trabajadores sobre las tendencias actuales de su especialidad en los servicios Premium.

2. Confeccionar ficha de costo promedio por cliente del servicio Premium.

3. Crear alianzas estratégicas con nuevos turoperadores y agencias de viaje que vendan paquetes a clientes de lujo como: Pegas Touristik, CIC Colombia, Havanatur Chile y Argentina.

4. Identificar las líneas aéreas que conectan los mercados meta con el destino Holguín.

5. Diseñar un catálogo específico del servicio Premium para la red de ventas.

6. Actualizar los atractivos y ofertas del servicio Premium y del entorno en el sitio web del hotel mediante la interacción con los clientes. 
7. Gestionar la reputación online del servicio Premium en el sitio web del hotel, en el sitio web de Gaviota S. A y en las redes sociales (Tripadvisor, Zoover, Monarc, Holidaycheck).

8. Ampliar las negociaciones a través del MINTUR con los turoperadores rusos.

Tarea 11. Evaluación de indicadores

En la operación del servicio Premium hasta el 2017 puede apreciarse un incremento por año: así, en el 2015 con un precio medio de 26,95 CUC y sólo 6588 clientes/ días se pudo obtener un ingreso de 177 mil CUC; en el 2016 se apreció un discreto aumento del precio medio, que fue de 56,59 CUC, lográndose 11421 clientes/ días, para un ingreso total de 646 mil CUC; por último, en el 2017, los clientes/ días fueron 13754 con un precio medio de 82,91 CUC, lo que permitió obtener un ingreso de 1140 mil CUC, de ellos 494 mil CUC por encima del año 2016, ver la tabla siguiente:

Tabla N4. Análisis de los indicadores Clientes/Días, Ingreso e Ingreso medio/Clientes/ Días

\begin{tabular}{|c|c|c|c|}
\hline Año & Clientes/ Días & $\begin{array}{c}\text { Ingreso } \\
\text { (CUC) }\end{array}$ & $\begin{array}{c}\text { Ingreso medio/ Clientes/ Días } \\
\text { (CUC) }\end{array}$ \\
\hline 2015 & 6588 & 177594,03 & 26,95 \\
\hline 2016 & 11421 & 646359,72 & 56,59 \\
\hline 2017 & 13754 & 1140435,86 & 82,91 \\
\hline
\end{tabular}

Fuente: Elaboración Propia

De un comportamiento negativo en la comercialización del servicio en su primer año 2015 se evolucionó favorablemente, lo que permitió en 2017 un incremento del nivel de ocupación de un 35,89\%.

Valoración de la Estrategia para la comercialización del servicio Premium

A través del criterio de especialistas se valoró la Estrategia para la comercialización del servicio Premium en del hotel Playa Pesquero, para lo cual fue necesario:

1. Evaluar el nivel de conocimiento de los especialistas;

2. Elaborar un test sobre las líneas estratégicas relacionadas con las acciones de implantación de las estrategias de producto, precio, distribución y promoción;

3. Procesar los resultados a partir de las respuestas emitidas por los especialistas.

Fueron seleccionados 15 especialistas vinculados al Grupo de Turismo Gaviota S. A., de ellos ocho poseían un nivel de conocimiento alto, y dos de nivel medio, por lo que el resto fue descartado. A los 10 especialistas seleccionados se les aplicó el test, para lo cual sólo fue necesario realizar una ronda, dado el alto consenso alcanzado en los cinco aspectos consultados, así, la propuesta de las estrategias producto, precio, distribución y promoción 
lograron una alta pertinencia para un 100\%, sin embargo, con respecto a la aplicabilidad de las estrategias el $77 \%$ consideró alta la aplicabilidad y solamente un $23 \%$ le otorgó un valor medio, entre otras causas, porque vender todo el año con el precio de contrato sin hacer oferta resulta muy difícil por los cambios que surgen en los mercados, además, todos los segmentos no reaccionan de la misma manera, lo que dificulta la obtención a mediano plazo de los resultados deseados.

Dentro de las sugerencias de acciones propuestas por los especialistas consultados se encuentran las siguientes:

1. Renovar los precios de contrato con principales turoperadores con los que opera el hotel (Thomas Cook, Transat y Sunwing) para venderlo todo el año;

2. Utilizar la sección Premium para el hospedaje de grupos de incentivos con alto poder adquisitivo;

3. Desarrollar nuevos paquetes de luna de miel y bodas;

4. Lograr que el servicio Premium sea la escogida por las AA.VV receptivas Havanatur, Viajes Cubanacán y Gaviotatur para los overnight de opcionales en el territorio, para clientes de alto poder adquisitivo;

5. Realizar publicaciones en medios digitales como Caribbean News Digital;

6. Construir un snack en el área de la playa.

La estrategia aplicada contribuye, desde el servicio Premium, al cumplimiento de los aspectos relacionados con: la elevación de la calidad de los servicios y el logro de una adecuada coherencia en la relación calidad/precio; el perfeccionamiento de las formas de comercialización mediante el uso de las tecnologías más avanzadas de la información y las comunicaciones; y el incremento de los arribos, al diversificar los mercados emisores y los segmentos de clientes, elevando los ritmos de crecimiento en correspondencia con el desarrollo turístico.

Finalmente, la aplicación parcial de la estrategia permitió la transformación de la problemática identificada en el servicio Premium y el logro de resultados favorables en su comercialización, se logra así dar respuesta al problema científico identificado, por lo que se cumple la idea a defender en la investigación.

\section{CONCLUSION}

1. La estrategia diseñada para la comercialización del servicio Premium en el hotel Playa Pesquero posee cuatro componentes esenciales: fases, objetivos, tareas y técnicas a emplear. Se distingue por incluir el diseño del perfil de los mercados meta, las 
potencialidades del producto para esos respectivos mercados y proporciona herramientas para confeccionar las estrategias producto, precio, distribución y promoción.

2. La estrategia para la comercialización del servicio Premium en el hotel Playa Pesquero posee cinco líneas estratégicas dirigidas a: Identificar y trabajar por la incorporación de segmentos de clientes en los mercados actuales de Canadá, Reino Unido, Alemania e Italia; Captar segmentos de clientes procedentes de los mercados potenciales: Rusia, Polonia, Austria, Chile y Estados Unidos; Identificar y trabajar por la incorporación de segmentos de clientes de negocios, eventos y diplomáticos en Cuba y en los mercados actuales del hotel; y por último, Establecer y vender todo el año el servicio Premium con los precios de contrato con los turoperadores.

3. La aplicación parcial de la estrategia para la comercialización del servicio Premium en el hotel Playa Pesquero obtuvo entre sus resultados fundamentales el diseño del perfil de los mercados meta: Canadá, Reino Unido, Alemania e Italia; la actualización de los objetivos estratégicos; el diseño de las estrategias de producto, precio, distribución y promoción; así como el incremento de los ingresos del servicio, lo que valida la idea a defender y resuelve el problema que generó la investigación.

\section{REFERENCIAS}

Beauchamp M.; Barnes, D. (2015). Delighting Baby Boomers and Millennials: Factors that Matter Most. Journal of Marketing Theory and Practice. Vol. 23 (3), pp. 338-350.

Cabrisas Ricardo. (2017). "Discurso ante la Asamblea Nacional”. [En línea]. Disponible en: http://www.cubadebate.cu/noticias Consultado el 30 de diciembre de 2017

Cardet Fernández, E. (2007). Modelo sobre diseño de estrategias en el área de ciencia e innovación tecnológica. (Tesis en opción al título académico de Master en Gestión Turística). Universidad de Holguín. Holguín, Cuba.

Córdova, J. L. (2016). Tendencias tecnológicas en turismo para el 2016. [Mensaje en un Blog]. Thinktur. Plataforma tecnológica de Turismo. Recuperado de: https://www.thinktur.org/media/Ebook_Tendencias_Tec_Turismo_2016.pdf

De la Mora, E. (2017). Turismo de Lujo PREMIUM. Revista AyB. Recuperado de: http://www.revista-ayb.com/promocion-turistica/turismo-de-lujo.

De Miguel y Barrios, C. (2016). Tendencias tecnológicas en turismo para el 2016. [Mensaje en un Blog]. Thinktur. Plataforma tecnológica de Turismo. Recuperado de: https://www.thinktur.org/media/Ebook_Tendencias_Tec_Turismo_2016.pdf

Escalona Betancourt R. (2010). Procedimiento para la formulación de estrategias de un mercado emisor de un destino. (Tesis en opción al título académico de Master en Gestión Turística). Universidad de Holguín. Holguín, Cuba. 
Figueiras Rodríguez, J. (2018). Estrategia para la comercialización del servicio Premium del hotel Playa Pesquero. (Tesis en opción al título académico de Máster en Gestión Turística). Facultad de Ciencias Empresariales y Administración. Universidad de Holguín. Holguín, Cuba.

Guerrero G. (2014). 7 Claves para identificar un producto o servicio Premium. [Mensaje en un Blog]. Mercadotecnia Alternativa. Recuperado de: www.gustavoguerrero.me/2014/01/7-claves-para-identificar-un-producto-o.html

Guiñazu. J. A. (2016). Las características de los hoteles de lujo. Articulo TV. Recuperado de: http://www.articulo.tv/Las-caracteristicas-hoteles-lujo_8851

Harmon, M. (01 de enero 2016). Experiencias del cliente en hoteles: tendencias para 2016. Hosteltur. Recuperado de: https://www.hosteltur.com/114402_experiencia-clientehoteles-tendencias-2016.html

Hernández, Y. (2010). Procedimiento para la elaboración e implementación del plan de marketing en pequeños y medianos hoteles de tránsito del destino Villa Clara. (Tesis presentada en opción al título académico de Master en Ciencias). Santa Clara, Centro de Estudios Turísticos, Universidad Marta Abreu de Las Villas. Santa Clara, Cuba.

Laico, F. (2017). Revenue management y hoteles cinco estrellas. Revenue para hoteles. Recuperado de: https://www.revenueparahoteles.com/revenue-management-yhoteles-de-5-estrellas/

Larraiza, L. (2017) Clasificación de Hoteles. [Mensaje en un Blog]. El Blog de Leire Larraiza. Tendencias sobre hotelería y Turismo. Disponible en: https://leirelarraiza.com/calidad/clasificacion-de-hoteles.

Marrero, M. (07 de Junio de 2017). Inauguran primer hotel cubano de lujo cinco estrellas plus. Granma. Recuperado de: http://www.granma.cu/cuba/2017-06-07/inauguranprimer-hotel-cubano-de-lujo-cinco-estrellas-plus-07-06-2017-23-06-27

Medina Labrada, J. (2012). Estrategia comercial del destino turístico de Holguín para el mercado Ruso. (Tesis en opción al título académico de Master en Gestión Turística). Universidad de Holguín. Holguín, Cuba.

Miralles P. (2016). Tendencias tecnológicas en turismo para el 2016. [Mensaje en un Blog]. Thinktur. Plataforma tecnológica de Turismo. Recuperado de: https://www.thinktur.org/media/Ebook_Tendencias_Tec_Turismo_2016.pdf

Palao Fuentes, R. (2001). Procedimiento para la elaboración de un plan de marketing para un destino turístico. (Tesis en opción al título académico de Máster en Dirección). Universidad de Holguín. Holguín, Cuba.

Peña Hernández M. (2006). Procedimiento para posicionar el destino Holguín en los

Visión de Futuro" Año 16, Volumen Nº 23 N², Julio - Diciembre 2019 - Pág. 252 - 273

URL de la Revista: http://visiondefuturo.fce.unam.edu.ar/index.php/visiondefuturo/index

URL del Documento: http://visiondefuturo.fce.unam.edu.ar/index.php/visiondefuturo/issue/view/16 
mercados emisores que lo visitan. (Tesis en opción al título académico de Master en Gestión Turística). Universidad de Holguín. Holguín, Cuba.

Pérez Porto, J y Merino M. (2015.) Definición de Premium. Definicion.de. Recuperado de: https://definicion.de/premium/

Rivero Rey R. (2008). Procedimiento para la gestión comercial de un destino turístico. (Tesis en opción al título académico de Master en Gestión Turística). Universidad de Holguín. Holguín, Cuba.

Rodríguez Pérez M. (2008). Metodología para la planificación estratégica en el área comercial. (Tesis en opción al título académico de Máster en Gestión Turística). Universidad de Holguín. Holguín, Cuba.

Ruz, A. (2017). Qué características de higiene y limpieza deben reunir un hotel cinco estrellas. [Mensaje en un Blog]. Viajero Turismo. Recuperado de: http://www.viajeroturismo.com/2017/12/que-caracteristicas-de-higiene-y.html

Stoner J. A. F, Freeman B. E y Gilbert Jr. D. R. (1996). Management. Sexta Edición. México. Prentice Hall.

Vieytes, Maritza. (2010). Técnica de Escenarios. Disponible en: http:// www4.ujaen.es/ egarcia/programadedirest05-06.doc

\section{RESUMEN BIOGRAFICO}

\section{Justa Medina Labrada}

Arquitecta (1984), Universidad de Oriente. Especialista de Desarrollo, Delegación del Ministerio de Turismo- Holguín (2001). Máster en Gestión Comercial por la ESEM de Madrid (2002). Máster en Gestión Turística en Universidad de Holguín (2012). Profesora Auxiliar del Departamento de Turismo, Universidad de Holguín desde 2007. Dirige investigaciones sobre marketing organizacional.

\section{Migdely Barbarita Ochoa Avila}

Ingeniera Química (1993), Universidad de Camagüey, Cuba. Doctora en Ciencias Técnicas del Instituto de Tecnología y Ciencias Aplicadas de Cuba (2014). Profesora Titular y Secretaria del Consejo Científico de Facultad de Ciencias Empresariales en Universidad de Holguín, Cuba. Profesora del Departamento de Turismo (2014). Dirige investigaciones de gestión organizacional.

\section{José L. Figueiras Rodríguez}

Licenciado en Economía (2001), Universidad de Holguín, Cuba. Especialista de la Compañía Gaviota S.A. desde 2001-2003. Jefe de Departamento de Riesgos desde 2003 en el Hotel Playa Pesquero, Provincia de Holguín. Máster en Gestión Turística por la Facultad de Ciencias Empresariales en Universidad de Holguín, 2018. 